\title{
CURRENT UNDERSTANDING ON DIVERSITIES OF GUT MICROBIOTA IN WILD AND DOMESTIC ANIMALS AND IMPLICATION OF THE KNOWLEDGE IN ANIMAL HEALTH AND PRODUCTION
}

\author{
H. K. MAITY ${ }^{1}$, S. BISWAS ${ }^{1}$, S. DAS $^{2}$, P. SREE LAKSHMI ${ }^{3 \#}$ AND I. SAMANTA $^{4 *}$ \\ ${ }^{1}$ Department of Avian Science, West Bengal University of Animal and Fishery Sciences, Mohanpur \\ Campus, Nadia-741 252, West Bengal, India \\ ${ }^{2}$ Department of Fish Nutrition,West Bengal University of Animal and Fishery Sciences, Chakgaria \\ Campus, Kolkata-700 094, West Bengal, India \\ ${ }^{3}$ College of Veterinary and Animal Sciences, Kerala Veterinary and Animal Sciences University, \\ Pookode, Wayanad - 673 576, Kerala, India \\ ${ }^{4}$ Department of Veterinary Microbiology, West Bengal University of Animal and Fishery Sciences, \\ Belgachia, Kolkata-700 037, West Bengal, India
}

\begin{abstract}
The scientific world became enthusiastic to develop the concept of 'gut health', 'intestinal health', and 'enteric health' specially after the banning of antibiotic growth promoter by European Union in poultry diet. The composition and functionality of the gut microbiome is crucial for the health of livestock and poultry as it helps in the development of intestinal epithelium and modulation of the physiological functions. Thus the intestinal homeostasis i.e. immunity, nutrient digestion, intestinal barrier integrity is maintained. The overall composition of microorganisms dwelling in the gastrointestinal tract is termed as 'microbiome' and due to its beneficial effects on health and well-being of host it has gained lot of attention in recent years. The present review describes gut microbiome composition in livestock and wild animals, effect of host genotype, human interference, environment on the composition of gut microbiome, detection techniques of gut microbiome composition, and implication and use of probiotics improving health and production in domestic animals.
\end{abstract}

Key words: Gut, Livestock, Microbiome, Poultry, Wild animals

\section{Introduction}

During last decade the scientific world became enthusiastic to develop the concept of 'gut health', 'intestinal health', and 'enteric health' especially after the banning of antibiotic growth promoter (AGP) by European Union in poultry diet (Castanon, 2007). Hippocrates (400 B.C.) was the first person to believe that 'all disease begins in the gut' and 'death sits in the bowel'. Gut health is difficult to define precisely. It is considered as the ability of the gut to perform normal physiological functions and to maintain homeostasis, thereby supporting its ability to withstand infectious and non-infectious stressors (Kogut et al., 2017). It is a holistic approach associated with nutrition, gut physiology, microbiology and immunology. The composition and functionality of the gut microbiome is crucial for the health of livestock 
and poultry as it helps in the development of intestinal epithelium and modulation of the physiological functions (Diaz Carrasco et al., 2019). Thus the intestinal homeostasis i.e. immunity, nutrient digestion, and intestinal barrier integrity is maintained. Like other animals, the livestock and poultry also naturally exposed to the ocean of microbes through air, feed and water after birth. A 'healthy' animal or bird can be defined as the animals which can meet the performance standard with normal physiological functions but essentially not 'germ-free' (Broom, 2011). The genome of all microbes inhabiting in the gut is termed as 'gut microbiome' and due to its beneficial effect on health and well-being of host it has gained lot of attention in recent years (Kogut, 2019). The present review describes gut microbiome composition in livestock and wild animals, effect of host genotype, human interference, environmental stress on the composition of gut microbiome, detection techniques of gut microbiome composition, and implication and use of probiotics improving health and production in domestic animals.

\section{General overview of microbiome composition of domestics and wild animals}

The diverse population of gut microbiota has important role in host metabolism, immunity, production, and prevention of infections. Hostgut microbiota is a complex system and numerous studies showed imbalance of gut microbiome is often associated with metabolic disorders and pathogen resistance. With the modern standards of living most population in the developed countries of the world showed lower gut microbial diversity compared with population living in simple life style such as eastern Africa or Amazonian forest, who have not been exposed to antibiotics and modern lifestyles (Fan and Pedersen, 2020). The gradual decline in the Bacteroides, Prevotella, Desulfovibrio, Lactobacillus and Oxalobacteris among the gut microbiota often associated with chronic metabolic disorder in urban population. Studies showed that germ-free mice have thinner intestinal lining and underdeveloped lymphoid tissue, less nutrient absorption capability as well as low immune response compared to wild mice (Round and Mazmanian, 2009). The diverse range of gut microbiota creates a complex host microbiome relationship in animals impacting metabolic and immune response of the host ( $\mathrm{Xu}$ et al., 2013). The factors influencing gut microbial diversity depends on complex web of factors, such as, species, age, geographical distribution, nutritional recourses, environmental and anthropogenic factors, which can be summarised into two major categories as endogenous and exogenous factors (Wang et al., 2018). The factors related to host genetics considered as endogenous, whereas factor related to difference in feed composition and environmental factors are major exogenous factors regulating gut microbial diversity (Knowles et al., 2019). Understanding of complex gut microbiome relationship and underlying causes of diversity of microbiome among domestic and wild animal would be important for animal production, immunity, maintenance of health and conservation of wild animal.

Gut microbiome produces the large variety of bioactive compounds by using host ingested feed or interacting with host derived cells, which plays an important role in host metabolism (Tanca et al., 2017). The major composition of gut microbiome in vertebrates is dominated by bacterial phylum Firmicutes, Bacteroidetes, Actinobacteria, Fusobacteria although the distribution differs across the species (Dearing and Kohl, 2017). The herbivore gastro intestinal (GI) tracts are mostly dominated by Firmicutes and Bacteroidetes due to their precise role in cellulose metabolism (Muegge et al., 2011). 
Diverse population of gut microbial community improves the digestive efficacy of complex polysaccharides, which otherwise is inaccessible to animal metabolism. Alteration in gut microbiome may disturb the digestive metabolite, which is beneficial to host. Many studies showed that relative abundance of bacterial operational taxonomic units (OTU) was comparatively higher in wild animals than captive or domestic animals (Kohl and Dearing, 2016; McKenzie et al., 2017). The major bacterial community depleted in domestic animals belong to families such as Ruminococcaceae and Lachnospiraceae. Both the bacteria have glycoside hydrolase gene, known for metabolism of complex plant components like cellulose, hemicellulose and polysaccharides (Blank et al., 2012).

\section{Effect of host genetics in gut microbiome composition}

A significant variation can be observed on gut microbial composition across the species. Additionally, microbiota also showed variation between individuals within the same group. The large and small pig population have been studied as a model of human microbiome and in both the groups bacteria under phyla Firmicutes and Bacteroides predominates in GI tract (Lamendella et al., 2011; Pedersen et al., 2013). Dogs also have gut microbiota similar to humans, but diverge fundamentally in specific genera and comparative abundance of different phyla (Handl et al., 2011; Hoffmann et al., 2016). In wild macaques, markedly different gut microbiome compared to both the human and mouse, including complete absence of some major genera was detected (McKenna et al., 2008; Yildirim et al., 2010). The gut microbiota of black-and-white colobus monkey and red-tailed guenon are predominantly under phyla Firmicutes and Bacteroides (Barelli et al., 2015). The gut microbiome of Udzungwa red colobus monkey (Procolobus gordonorum) includes very few $(<0.01 \%)$ Fibrobacteres,
Actinobacteria and TM7 phylotypes, whereas Verrucomicrobia, Spirochetes and Proteobacteria were found significantly (Barelli et al., 2015). Ruminococcaceae and Lachnospiraceae families that are known to be highly specialized in the degradation of plant polysaccharides commonly consumed by ruminants are rich in xylan, pectin, and cellulose (Flint and Bayer, 2008).

The predominant microbiota found in poultry (chicken and turkey) are Firmicutes, Bateroidetes and Proteobacteria, which constitutes more than $90 \%$ of the operational taxonomic units (OTUs) (Wei et al., 2013). In a recent study on migratory water birds such as great cormorants (Phalacrocorax carbo), little egrets (Egretta garzetta), black-crowned night herons (Nycticorax nycticorax) and blackheaded gulls (Chroicocephalus ridibundus) showed that Fusobacteria, Firmicutes and Proteobacteria are dominant phyla among the gut microbes (Laviad-Shitrit et al., 2019). Fusobacteria, an obligate anaerobic non-spore forming Gram-negative bacilli, is considered as one of the most dominant phylum $(>34 \%)$ in all wild water bird species compared to other avian species (Laviad-Shitrit et al., 2019). In black headed gulls, Catellicoccus (a facultative anaerobic, Gram-positive bacteria) found to be the most abundant genus (58.86\%) (Lawson et al., 2006; Laviad-Shitrit et al., 2019). Interestingly, the study also showed that gut microbiota of migratory water birds also act as carrier of major human pathogens such as F. Necrophorum (human oral infections) and F. Nucleatum (human inflammatory bowel disease, liver cirrhosis) (Qin et al., 2014). Some other potential pathogenic bacteria found in gut microbiota of water birds are Clostridium, Helicobactor, Campylobactor, Arcobactor and Vibrio (Laviad-Shitrit et al., 2019). The studies thus indicate that migratory water birds can disburse potentially pathogenic bacterial species across intercontinental borders. 


\section{Human interference impacting gut microbiota of wild animals}

Anthropogenic factors have pronounced effect on the environment as well as other species on earth. Due to increased demand for supply of livestock, the number of domestic animals has increased, which correlates with the degradation of natural habitat and replacement with agricultural lands (McDonald et al., 2019). It directly creates scarcity of nutritional recourses for wild animals and the wild animals face higher selection pressure leading to massive change in their feeding habit and gut microbial diversity. Due to human interference domestic animals are fed with higher quality feed, which creates artificial scarcity of feed and leads to diversity in gut microbiota in wild animals particularly in a region, where wild animal share the similar nutritional resources with livestock (Li et al., 2013). For example, Qaidam Basin is a deserted area with vegetation coverage of less than $5 \%$ located at northern Qinghai Province in China. Wild goitered gazelles (Gazella subgutturosa) are main wild habitat, whereas sheep (Ovis aries) are major domestic animal of this land (Zhong et al., 2014). Due to human interference sheep are fed with higher quality of forage and goitered gazelles became forced to change their organ size to adopt with food shortages (Blank et al., 2015). A recent gut microbial analysis between wild goitered gazelles and domestic sheep showed the presence of Firmicutes $(76.40 \%$; $71.03 \%)$ and Bacteroides (17.17\%; 21.84\%) associated with cellulose and carbohydrate digestion in both the goitered gazelles and sheep (Qin et al., 2020). The same study also showed that Firmicutes/ Bacteroides ratio was comparatively higher in goitered gazelles than sheep. In addition Thaumarchaeota, Synergistetes, Chlorobi, and TM6 were only identified in goitered gazelles (Qin et al., 2020). In comparison, pathogenic bacteria such as Campylobacter, Helicobacter and Shigella spp. in the sheep were significantly higher than that of the goitered gazelles (Qin et al., 2020). It indicates that due to shortage of nutritional resources goitered gazelles might have adopted microbial composition for effective metabolism of poorer pasture, and the richness in the diversity of gut microbiome also reduced the pathogen load (Qin et al., 2020).

Many endangered species were introduced in captivity for effective breeding and conservation. Research on captive animals showed that during captivity, gut microbiome shifts dramatically due to limitation of geographical range, variation of feeds and controlled social interaction during captivity as compared to wild animals (Gibson et al., 2019). Many studies have shown that microbial diversity reduced in wild animal during captivity (Borbon-Garcia et al., 2017; McKenzie et al., 2017). In a recent study diversity of gut microbial composition of wild Indian bison (Bos gaurus), captive bison and domestic form of gour, known as Mithun (Bos frontalis) was compared (Prabhu et al., 2020). The study showed that the diversity of gut microbiome is lower in domestic and captive animals than wild animals. The study also showed that the content of cyanobacterial population, which ferments starch content of feed into anoxic condition, is higher in domestic and captive animals compared to the wild animals. It indicates that due to co-habitation around human vicinity, the gut microbial diversity of domestic animals probably adopted with higher cyanobacterial content to metabolise higher starch based feed (Prabhu et al., 2020). Higher cyanobacterial content was also observed in domestic dogs (Axelsson et al., 2013). Similar gut microbial difference was also detected in rhinoceros (Rhinoceros unicornis), where microbial richness was higher in wild rhino than captive rhinoceros, and gut microbiome replaced by microbe found in conventional domestic animal such as higher contents of Bacteriodetes and lower contents of Proteobacteria (Gibson et al., 2019). Disturbance of gut microbiome 
could also be linked with application of antibiotics and drugs, which is often associated with imbalance of gut microbiota and subsequent reduction of immunity in animals (Park, 2018).

\section{Role of animal feed in gut microbiome composition}

Impact of microbial communities on their host has increased many folds in recent years. The microbiome provides nutrition to the host and helps in development of immunity and metabolism (Backhed et al., 2005; Andreote and Pereira, 2017). In cattle, the research has conducted on microbiome manipulation to reduce methane emission in the environment. Digestion of plant materials (forage, fodder and hay) by methanogenic bacteria in the ruminant's intestines emits methane, which is responsible for climate change. Changing the microbiome community of rumen through addition of Methanobrevibacter reduces the methane emission (Danielsson et al., 2017). Microbial community of calves' changes rapidly after birth and at $12^{\text {th }}$ weeks of age Bacteroides prevotella, Clostridium coccoide and Eubacterium rectale predominates in the digestive system (Uyeno et al., 2010a, 2010b). At weaning, Bacteroidetes decreased, while Proteobacteria and Firmicutes increased in calves (Meale et al., 2016).

The digesta of foregut and hindgut in cattle mainly comprises of Gram-negative Bacteroidetes and the Gram-positive Firmicutes and together represents $76-96.1 \%$ of total bacteria (Plaizier et al., 2016). Feeding of high-grain diets may lead to subacute ruminal acidosis (SARA), a reversible metabolic disorder, characterized by lowering rumen $\mathrm{pH}$ for a variable period in each day. If the cattle consume high amount of grains in a short period of time, the Bacteroidetes and Fibrobacter group of bacteria decreases very rapidly and Firmicutes and other opportunistic bacteria (Gramnegative Proteobacteria) increases, resulting a dysbiotic community with loss of rumen function
(Khafipour et al., 2009). Bacteroidetes and Fibrobacter group of bacteria is the major contributor of free lipopolysaccharide (LPS) in the hindgut and rumen. The LPS acts as the potent endotoxin and is highly immunogenic (Hurley, 1995). Feeding high-grain diets also decreases the populations of ciliate protozoa (Khafipour et al., 2009; Plaizier et al., 2016). It seems that diet/food is the main feature in shaping the rumen microbiome (Henderson et al., 2015) and the arrangement of the core members of this microbial community is quite stable in adult animals (Petri et al., 2012).

Ceca, an integral part of GI tract of poultry, hold the highest microbial mass and accomplish most of the fermentation activities. Firmicutes, Bacteroides and Proteobacteria (clostridial species) are mainly found in the ceca of chicken (Oakley et al., 2014). Supplementation of xylo-oligosaccharides, degradation products of lignocellulose materials, soybean meal oligosaccharides, Lactobacillus and Bifido-bacterium in the diet increases the production of short chain fatty acids (SCFAs), which improves the gut health of poultry and nutritional quality of chickens (Brugman et al., 2018).

Dog is the closest companion animals to humans and in many parts of the world they are treated as family members. Dogs are mostly raised on natural food or commercial food. Dogs maintained on natural diet have $30-50 \%$ more chance to get infected with Salmonella and E. coli than that of commercial food (Kim et al., 2017). Clostridium perfringens and Fusobacterium varium are potent opportunistic pathogens found more commonly in the faeces of naturally maintained dogs and imposes greater risk of zoonotic hazards (Kim et al., 2017). However, large scale use of growth promoters or antibiotics in food animals and companion animals results dysbiotic relationship between microbiome and the host. 


\section{Role of environment in gut microbiome composition}

Environmental factors are one of the most important exogenous factors for influencing gut microbiome diversity. Various environmental factors such as temperature, geographical location affects gut microbiome of domestic and wild inhabitants. The gut microbiome refers to the particular population of microorganisms inhabiting a well-defined environment throughout the gastrointestinal tract, and includes bacteria, fungi, protozoa, archaea, and yeasts. GI microbiome is strongly influenced by micro and major environment. The GI microbiome of laboratory rodents changes, when exposed to various chemicals, heavy metals and ionizing radiation (Claus et al., 2016; Zhang and Steen, 2017). Few studies also reported how anthropogenic habitat alterations affect microbiome composition in wild animals (Lavrinienko et al., 2018). The difference of gut microbiome of Udzungwa red colobus monkey (Procolobus gordonorum) from other nonhuman primates related to plant diversity, degraded natural habitats and termite consumption during rainy season (Barelli et al., 2015). The relationship suggests a horizontal transfer of gut microbiome between prey and predator. Environmental parameters like temperature, humidity or precipitation are probably the potential causes for variation of gut microbiome in same species of animals residing in two different geographical locations (Barelli et al., 2015). Gut microbiome also vary in its composition and function, when animals placed in captive condition and free ranging condition. In animals, particularly in ruminants, wide variety of microbiome and its complex relationship with host coincides with environmental factors. The enteric microbiota plays an important role in digestion of nutrient and its assimilation, synthesis of volatile fatty acids (VFA), vitamins and amino acids (Turner, 2018). In animals, the alternation of composition of gut microbiota have related to different disease conditions and surrounding environmental conditions.

\section{Detection of gut microbiota composition}

The autochthonous microflora of the gastrointestinal tract is a rich ecosystem and it is a cumbersome procedure to identify and determine the variety of organisms. Traditional culture based techniques and nucleic acid based methods are the two major practices, which can provide a comprehensive data on gut microbiota. Microbial culture is considered to be gold standard as the specificity of the culture is $100 \%$ but the sensitivity remains low (Limmathurotsakul et al., 2010). The major demerit of microbial culture technique is its inability to detect many of the significant gut microbiota due to the limitations of substrates, media used for culture and a labour intensive procedure. Only less than $30 \%$ bacteria could be cultured till date and hence there is a need to revolutionize the knowledge with wide application of genome based techniques (Ignys et al., 2014).

The faeces of animals and humans are the choice of specimen for analysing the gut microflora. Both longitudinal and cross sectional studies can be done to assess the pathophysiology of gastric and hepatic diseases using faeces as a specimen (Allaband et al., 2019). The mucosal biopsy samples can be used in conjunction with faecal samples for a snapshot analysis of the gut microflora as the faeces fails to recognise many mucosal adherent floras (Eckburg et al., 2005).

The outcome is favourable, when the culturedependent analyses and culture-independent, nucleic acids-based techniques are done simultaneously. Genomic analysis employing polymerase chain reaction (PCR) for amplification of the 16S rRNA can provide an authentic representative data on gastrointestinal microflora as it is highly conserved among the bacterial species. The quantitative PCR can be used for the amplification as well as quantification of the 16S rRNA, which enables phylogenetic identification. Fluorescence in situ hybridization (FISH) and DNA microarray 
methods are employed for phylogenetic identification of the microbiota. Although a tedious process, DNA sequencing is considered as the gold standard tool for diagnosis as it can identify the species up to the taxonomical level. Direct sequencing of 16S rRNA gene amplicons using massive parallel sequencing incorporating pyro sequencer can be done to detect the microbiota at the phylogenetic level. It also helps to quantify the microflora and enables the identification of unknown bacteria (Fraher et al., 2012). The pros and cons of molecular techniques are described in Table 1.

Next-Generation Sequencing (NGS) is the latest tool for molecular genomic analysis, which allows the sequencing of whole microbial genome. Microbiome shotgun sequencing and metagenomics has revolutionized the field for gut microbiota analyses by establishing a correlation between clinical condition and the pattern of gut microbiota. Microbiome shotgun sequencing involves massive parallel sequencing of the mixed DNA sample. Shotgun sequencing employs random fragmentation of DNA followed by sequencing the fragments and reconstructing the overlapping sequences in order to assemble them into a continuous sequence (Jovel et al., 2016).
Implication and use of probiotics in improving health and production in domestic animals

The gut microbiome forms a protective barrier with the attachment of epithelial cells and thus prevents the attachment of pathogenic bacteria through 'competitive exclusion' (Yegani and Korver, 2008). The bacterial group can synthesize vitamins ( $\mathrm{K}$ and $\mathrm{B}$ complex), short chain fatty acids (SCFA) from polysaccharides, organic acids (lactic acid), antimicrobial compounds (bacteriocin) and produce non-specific immunity offering protection to the livestock and poultry (Apajalahti, 2005). The SCFA are readily absorbable form of polysaccharides generated by the gut microbes. The poultry do not have any metabolic cycle to generate such absorbable form of nutrients. However the microbiome present in caeca can breakdown the carbohydrates with the enzymes normally absent in chickens (Sergeant et al., 2014). The SCFA can reduce $\mathrm{pH}$ of the intestine and inhibit the growth of acid-sensitive bacteria, inhibit bile catabolism with conversion to secondary bile acids, improve mineral absorption, and growth of epithelium and lamina propria (Christl et al., 1997). Similarly the microbiota also helps in digestion of proteins as the birds

Table 1. Molecular approach for identification and characterization of gut microbiome

\begin{tabular}{lll}
\hline \multicolumn{1}{c}{ Molecular techniques } & \multicolumn{1}{c}{ Advantages } & \multicolumn{1}{c}{ Disadvantages } \\
\hline 16S rRNA sequencing & $\begin{array}{l}\text { It can identify single } \\
\text { molecule of rRNA }\end{array}$ & $\begin{array}{l}\text { Biasness occurred in primers, } \\
\text { costly and time consuming }\end{array}$ \\
\hline Real-time PCR & $\begin{array}{l}\text { Quantification of } \\
\text { DNA }\end{array}$ & Costly \\
\hline $\begin{array}{l}\text { Denaturing gradient gel electrophoresis } \\
\text { (DGGE), Temperature gradient gel }\end{array}$ & $\begin{array}{l}\text { Amplicons are used } \\
\text { from sequencing }\end{array}$ & $\begin{array}{l}\text { Variations detected between } \\
\text { laboratories, data generated } \\
\text { electrophoresis (TGGE), Temporal } \\
\text { temperature gradient gel electrophoresis }\end{array}$ \\
$\begin{array}{l}\text { (TTGE), Terminal restriction fragment } \\
\text { length polymorphism (dT-RFLP),Single } \\
\text { strand conformation polymorphism } \\
\text { (SSCP) }\end{array}$ & & \\
\hline Fluorescence in situ hybridization (Fish) & Highly sensitive & Laborious \\
\hline
\end{tabular}


are deficient in enzymes for complete nitrogen metabolism although half of the nitrogen sources are converted into ammonia and is excreated. The microbiome has a definitive role in development of gut immune system as $\mathrm{B}$ and $\mathrm{T}$ lymphocytes and $\operatorname{IgA}$ is not detected in germ-free birds of 28 days age (Tizard 2017). The segmented filamentous bacteria and Bacteroides fragilis was detected to promote Th17 and Treg cells in the intestine (Caballero and Pamer, 2015). The diversity of the gut bacteria also helps in development of $\mathrm{T}$-cell receptors in the gut and spleen (Mwangi et al., 2010).

The zoonotic pathogens such as Campylobacter and Salmonella present as a part of the microbiome can be transmitted into human along with antibiotic resistance determinants (Zhou et al., 2012). Moreover, presence of gut microbiome can compromise the growth rate due to competition with the host for energy and protein, production of toxic metabolites, catabolization of bile acids decreasing fat digestibility of the birds (Gaskins et al., 2002). The birds use a substantial amount of energy to control the microbial population such as secretion of mucin and $\operatorname{IgA}$, which prevents intestinal penetration of the microbes (Suzuki and Nakajima, 2014).

The microbiome is changeable with diet, ingestion of antibiotics, and infection by pathogens. The supplementation of probiotics, prebiotics and synbiotics in the diet can modulate the microbiome (Pan and Yu, 2014). The effect of feeding probiotics in performance of poultry is extensively investigated. The dietary supplementation of Lactobacillus, Bacillus subtilis, Bacillus licheniformis and others effectively decreased the population of pathogenic bacteria such as Salmonella and E. coli in chicken intestine (Pan and Yu, 2014). Supplementation of prebiotics can inhibit the colonization of pathogenic bacteria, produce antimicrobial peptides, modify the gut morphology and stimulate adaptive immune system. Dietary intake of fructooligosaccharide (FOS), mannanoligosaccharide (MOS), xylooligosacc-haride (XOS), galactooli-gosaccharide (GOS) and soybean meal oligosaccharide (SMO) increased beneficial bacterial mass (Lactobacillus, Bifidobacterium) with reduction of pathogen load (Salmonella, E. coli), and up regulate ileal tolllike receptor (TLR) 2b, TLR4, IL-12 and IFN- $\gamma$ in chicken (Pourabedin and Zhao, 2015). Supplementation of yeast and yeast cell wall products also reduced Salmonella and produced better humoral immunity against Newcastle disease (Ghosh et al., 2012). Dietary supplementation of enzymes, plant-derived compounds, organic acids including butyric acid and mitigation of mycotoxin can also improve the gut health. Currently application of antimicrobial peptides (AMPs), antibodies (egg yolk), pattern recognition receptor (PRR) ligands, bacteriophages and vaccines are under consideration for modulation of gut microbiome (Kogut, 2019).

It can be prevented by application of in ovo feeding or post-hatch diets composed of vitamins (C and $\mathrm{E}$ ), minerals, amino acids (L-arginine, L-lysine, L-histidine, threonine) etc. In ovo feeding is practiced by injection of the supplements into amniotic cavity, yolk sac and air sac (Jha et al., 2019). Nutritional supplements injected into the amniotic cavity are deposited in the lungs and intestine due to the rhythmic respiratory movements of the late-term embryo. Currently these routes are evaluated for application of probiotics and prebiotics to modulate the gut microbiome (Jha et al., 2019).

\section{Conclusions}

In livestock and poultry it is evident that gut microbiome and immunity are two key regulators for productivity. The immune system remains functional on the basis of constant sensing of the gut microbes, and any alteration 
in the microbial composition may have longterm consequences as the host develops inflammatory or immune conditions having major impact on productivity. The composition and function of the microbiome is influenced by diets, environmental conditions, and use of antibiotics. The knowledge of gut microbial composition and the factors influencing the

\section{REFERENCE}

Allaband C, McDonald D, Vázquez-Baeza Y, Minich JJ, Tripathi A et al., 2019. Microbiome 101: studying, analyzing, and interpreting gut microbiome data for clinicians. Clin Gastroenterol Hepatol, 17: 218230, doi: 10.1016/j.cgh.2018.09.017

Andreote FD and Pereira ESMC, 2017. Microbial communities associated with plants: learning from nature to apply it in agriculture. Curr Opin Microbiol, 37: 29-34, doi: 10.1016/ j.mib.2017.03.011

Apajalahti J, 2005. Comparative gut microflora, metabolic challenges, and potential opportunities. J Appl Poult Res, 14: 444-453, doi: 10.1093/japr/14.2.444

Axelsson E, Ratnakumar A, Arendt ML, Maqbool K, Webster MT et al., 2013. The genomic signature of dog domestication reveals adaptation to a starch-rich diet. Nature, 495(7441): 360-364, doi: 10.1038 /nature 11837

Backhed F, Ley RE, Sonnenburg JL, Peterson DA and Gordon JI, 2005. Host bacterial mutualism in the human intestine. Science, 307: 1915-1920, doi: 10.1126/science. 1104816

Barelli C, Albanese D, Donati C, Pindo M, Dallago C et al., 2015. Habitat fragmentation is associated to gut microbiota diversity of an endangered primate: implications for conservation. Sci Rep, 5: 14862, doi: $10.1038 /$ srep14862

Blank D, Ruckstuhl K and Yang W, 2012. Influence of population density on group sizes in goitered gazelle (Gazella subgutturosa Guld., 1780). Eur J Wildl Res, 58: 981-989, doi: 10.1007/s10344012-0641-3

Blank DA, Ruckstuhl K and Yang W, 2015. Seasonal dynamics of agonistic displays in territorial and non-territorial males of goitered gazelle. Zoology, 118(1): 63-68, doi: 10.1016/j.zool.2014.08.004 composition in livestock and wild animals living in the same interface is getting crucial day by day. With the increasing evidence demonstrating the importance of the microbiota in maintaining intestinal homeostasis, it is likely that monitoring the composition of gut microbiome could be routinely included in the farms in the future.

Borbon-Garcia A, Reyes A, Vives-Florez M and Caballero S, 2017. Captivity shapes the gut microbiota of andean bears: insights into health surveillance. Front Microbiol, 8: 1316, doi: 10.3389/fmicb.2017.01316

Broom DM, 2011. A history of animal welfare science. Acta Biotheoretica, 59: 121-137, doi: 10.1111/soru. 12011

Brugman S, Ikeda-Ohtsubo W, Braber S, Folkerts G, Pieerse CMJ et al., 2018. A comparative review on microbiota manipulation: lessons from fish, plants, livestock and human research. Front Nutr, 5: 80, doi: 10.3389/fnut.2018.00080

Caballero S and Pamer EG, 2015. Microbiota-mediated inflammation and antimicrobial defense in the intestine. Annu Rev Immunol, 33: 227-256, doi: 10.1146/annurev-immunol-032713-120238

Castanon JIR, 2007. History of the use of antibiotic as growth promoters in European poultry feeds. Poult Sci, 86(11): 2466-2471, doi: 10.3382/ps.2007-00249

Christl SU, Bartram P, Paul A, Kelber E, Scheppach W et al., 1997. Bile acid metabolism by colonic bacteria in continuous culture: effects of starch and pH. Ann Nutr Metab, 41: 45-51, doi: 10.1159/ 000177977

Claus S, Guillou H and Ellero-Simatos S, 2016. The gut microbiota: A major player in the toxicity of environmental pollutants? npj Biofilms Microbiomes, 2: 16003, doi: 10.1038/ npjbiofilms.2016.3

Danielsson R, Dicksved J, Sun L, Gonda H, Muller B et al., 2017. Methane production in dairy cows correlates with rumen methanogenic and bacterial community structure. Front Microbiol, 8: 226, doi: $10.3389 /$ fmicb.2017.00226 
Dearing MD and Kohl KD, 2017. Beyond fermentation: other important services provided to endothermic herbivores by their gut microbiota. Int Comp Biol, 57: 723-731, doi: 10.1093/icb/icx020

Diaz Carrasco JM, Casanova NA and Fernández Miyakawa ME, 2019. Microbiota, gut health and chicken productivity: what is the connection? Microorganisms, 7 : 374, doi: $10.3390 \%$ 2Fmicroorganisms 7100374

Eckburg PB, Bik EM, Bernstein CN, Purdom E, Dethlefsen L et al., 2005. Diversity of the human intestinal microbial flora. Science, 308: 16351638, doi: 10.1126/science. 1110591

Fan Y and Pedersen O, 2020. Gut microbiota in human metabolic health and disease. Nat Rev Microbiol, 19(1): 55-71, doi: 10.1038/s41579-020-0433-9

Flint HJ and Bayer EA, 2008. Plant cell wall breakdown by anaerobic microorganisms from the mammalian digestive tract. Ann N Y Acad Sci, 1125: 280-288, doi: 10.1196/annals. 1419.022

Fraher MH, O'toole PW and Quigley EMM, 2012. Techniques used to characterize the gut microbiota: A guide for the clinician. Nat Rev Gastroenterol Hepatol, 9: 312-322, doi: 10.1038/ nrgastro.2012.44

Gaskins HR, Collier CT and Anderson DB, 2002. Antibiotics as growth promotants: mode of action. Anim Biotechnol, 13(1): 29-42, doi: 10.1081/ABIO-120005768

Ghosh TK, Haldar S, Bedford MR, Muthusami N and Samanta I, 2012. Assessment of yeast cell wall as replacements for antibiotic growth promoters in broiler diets: effects on performance, intestinal histo morphology and humoral immune responses. J Anim Physiol Anim Nutri, 96: 275284, doi: 10.1111/j.1439-0396.2011.01155.x

Gibson KM, Nguyen BN, Neumann LM, Miller M, Buss $\mathrm{P}$ et al., 2019. Gut microbiome differences between wild and captive black rhinoceros implications for rhino health. Sci Rep, 9: 7570, doi: $10.1038 / \mathrm{s} 41598-019-43875-3$

Handl S, Dowd SE, Garcia-Mazcorro JF, Steiner JM and Suchodolski JS, 2011. Massive parallel 16S rRNA gene pyrosequencing reveals highly diverse fecal bacterial and fungal communities in healthy dogs and cats. FEMS Microbol Ecol, 76(2): 301-
310, doi: 10.1111/j.1574-6941. 2011.01058.x

Henderson G, Cox F, Ganesh S, Jonker A and Young W, 2015 Global Rumen Census Collaborators, and P.H. Janssen. Rumen microbial community composition varies with diet and host, but a core microbiome is found across a wide geographical range. Sci Rep, 5: 14567, doi: $10.1038 /$ srep 14567

Hoffmann AR, Proctor LM, Surette MG and Suchodolski JS, 2016. The microbiome: the trillions of microorganisms that maintain health and cause disease in humans and companion animals. Vet Pathol, 53(1): 10-21, doi: 10.1177/ 0300985815595517

Hurley JC, 1995. Endotoxemia: methods of detection and clinical correlates. Clin Microbiol Rev, 8: 268-292, doi: 10.1128/CMR.8.2.268-292.1995

Ignys I, Szachta P, Galecka M, Schmidt M and PazgratPatan M, 2014. Methods of analysis of gut microorganism- actual state of knowledge. Ann Agri Environ Med, 21(4): 799-903, doi: 10.5604/ 12321966.1129936

Jha R, Singh AK, Yadav S, Berrocoso JFD and Mishra $\mathrm{B}, 2019$. Early nutrition programming (in ovo and post-hatch feeding) as a strategy to modulate gut health of poultry. Front Vet Sci, 6: 82, doi: 10.3389/fvets.2019.00082

Jovel J, Patterson J, Wang W, Hotte N, O'Keefe S et al, 2016. Characterization of the gut microbiome using $16 \mathrm{~S}$ or shotgun metagenomics. Front Microbiol, 20: 459, doi: 10.3389/fmicb. 2016.00459

Khafipour E, Li S, Plaizier JC and Krause DO, 2009. Rumen microbiome composition determined using two nutritional models of subacute ruminal acidosis. Appl Environ Microbiol, 75(22): 71157124, doi: 10.1128/AEM. 00739-09

Kim J, An JU, Kim W, Lee S and Cho S, 2017. Differences in the gut microbiota of dogs (Canis lupus familiaris) fed a natural diet or a commercial feed revealed by the Illumina MiSeq platform. Gut Pathog, 9: 68, doi: 10.1186/s13099-017-0218-5

Knowles SCL, Eccles RM and Baltrûnaitë L, 2019. Species identity dominates over environment in shaping the microbiota of small mammals. Ecol Lett, 22(5): 826-837, doi: 10.1111/ele.13240

Kogut MH, 2019. The effect of microbiome 
modulation on the intestinal health of poultry. Anim Feed Sci Tech, 250: 32-40, doi: 10.1016/j.anifeedsci.2018.10.008

Kogut MH, Yin X, Yuan J and Broom L, 2017. Gut health in poultry. CAB Rev, 12: 1-7, doi: 10.1079/PAVSNNR201712031

Kohl KD and Dearing MD, 2016. The woodrat gut microbiota as an experimental system for understanding microbial metabolism of dietary toxins. Front Microbiol, 7: 1165, doi: 10.3389/ fmicb.2016.01165

Lamendella R, Santo Domingo JW, Ghosh S, Martinson $\mathrm{J}$ and Oerther DB, 2011. Comparative fecal metagenomics unveils unique functional capacity of the swine gut. BMC Microbiol, 11: 103, doi: 10.1186/1471-2180-11-103

Laviad-Shitrit S, Izhaki I, Lalzar M and Halpern M, 2019. Comparative analysis of intestine microbiota of four wild waterbird species. Front Microbiol, 10: 1911, doi: 10.3389/fmicb .2019 .01911

Lavrinienko A, Tukalenko E, Mappes T and Watts PC, 2018. Skin and gut microbiomes of a wild mammal respond to different environmental cues. Microbiome, 6: 209, doi: 10.1186/s40168-018-0595-0

Lawson PA, Collins MD, Falsen E and Foster G, 2006. Catellicoccus marimammalium gen. nov., sp. nov., a novel Gram-positive, catalase-negative, coccusshaped bacterium from porpoise and grey seal. Int J Syst Evol Microbiol, 56: 429-432, doi: 10.1099/ijs.0.63874-0

Li XL, Gao J, Brierley G, Qiao YM, Zhang J et al., 2013. Rangeland degradation on the Qinghai-Tibet plateau: implications for rehabilitation. Land Degrad Dev, 24: 72-80, doi: 10.1002/ldr.1108

Limmathurotsakul D, Jamsen K, Arayawichanont A, Simpson JA, White LJ et al., 2010. Defining the true sensitivity of culture for the diagnosis of melioidosis using Bayesian latent class models. PLoS One, 5: e12485, doi: 10.1371/ journal.pone. 0012485

McDonald CK, Macleod ND, Lisson S and Corfield JP, 2019. The integrated analysis tool (Iat) - A model for the evaluation of crop-livestock and socio-economic interventions in smallholder farming systems. Agric Syst, 176: 102659, doi: 10.1016/j.agsy.2019.102659
McKenna P, Hoffmann C, Minkah N, Aye PP, Lackner A et al., 2008. The macaque gut microbiome in health, lentiviral infection, and chronic enterocolitis. PLoS Pathog, 4(2): e20, doi: 10.1371/journal.ppat.0040020

McKenzie VJ, Song SJ, Delsuc F, Prest TL, Oliverio AM et al., 2017. The effects of captivity on the mammalian gut microbiome. Integr Comp Biol, 57(4): 690-704, doi: 10.1093/icb/icx090

Meale SJ, Li S, Azevedo P, Derakhshani H, Plaizier JC et al., 2016. Development of ruminal and fecal microbiomes are affected by weaning but not weaning strategy in dairy calves. Front Microbiol, 7: 582, doi: 10.3389/fmicb.2016.00582

Muegge BD, Kuczynski J, Knights D, Clemente JC, González A et al., 2011. Diet drives convergence in gut microbiome functions across mammalian phylogeny and within humans. Science, 332: 970974, doi: 10.1126/science. 1198719

Mwangi WN, Beal RK, Powers C, Wu X, Humphrey T et al., 2010. Regional and global changes in TCRalphabeta $\mathrm{T}$ cell repertoires in the gut are dependent upon the complexity of the enteric microflora. Dev Comp Immunol, 34(4): 406-417, doi: 10.1016/j.dci.2009.11.009

Oakley BB, Lillehoj HS, Kogut MH, Kim WK, Maurer JJ et al., 2014. The chicken gastrointestinal microbiome. FEMS Microbiol Lett, 360(2): 100112, doi: 10.1111/1574-6968.12608

Pan D and Yu Z, 2014. Intestinal microbiome of poultry and its interaction with host and diet. Gut Microb, 5(1): 108-119, doi: 10.4161/gmic.26945

Park W, 2018. Gut microbiomes and their metabolites shape human and animal health. J Microbiol, 56: 151-153, doi: 10.1007/s12275-018-0577-8

Pedersen R, Andersen AD, Mølbak L, Stagsted J and Boye M, 2013. Changes in the gut microbiota of cloned and non-cloned control pigs during development of obesity: gut microbiota during development of obesity in cloned pigs. BMC Microbiol, 13(1): 30, doi: $10.1186 / 1471-2180-13-30$

Petri RM, Forster RJ, Yang W, McKinnon JJ and McAllister TA, 2012. Characterization of rumen bacterial diversity and fermentation parameters in concentrate fed cattle with and without forage. J Appl Microbiol, 112(6): 1152-1162, doi: 10.1111/j.1365-2672.2012. 05295.x 
Plaizier JC, Li S, Tun HM, Krause DO and Khafipour E, 2016. Nutritional models of experimentallyinduced subacute ruminal acidosis (SARA) differ in their impact on rumen and hindgut bacterial communities in dairy cows. Front Microbiol, 7: 2128, doi: 10.3389/fmicb.2016

Pourabedin M and Zhao X, 2015. Prebiotics and gut microbiota in chickens. FEMS Microbiol Lett, 362(15): fnv122, doi: 10.1093/femsle/fnv122

Prabhu VR, Wasimuddin, Kamalakkannan R, Arjun MS and Nagarajan M, 2020. Consequences of domestication on gut microbiome: A comparative study between wild gaur and domestic mithun. Front Microbiol, 11: 133, doi: 10.3389/fmicb.2020.00133

Qin N, Yang F, Li A, Prifti E, Chen Y et al., 2014. Alterations of the human gut microbiome in liver cirrhosis. Nature, 513: 59-64, doi: 10.1038/ nature 13568

Qin W, Song P, Lin G, Huang Y, Wang L et al., 2020. Gut microbiota plasticity influences the adaptability of wild and domestic animals in coinhabited areas. Front Microbiol, 11: 125, doi: 10.3389/fmicb.2020.00125

Round JL and Mazmanian S K, 2009. The gut microbiota shapes intestinal immune responses during health and disease. Nat Rev Immunol, 9: 313-323, doi: 10.1038/nri2515

Sergeant MJ, Constantinidou C, Cogan TA, Bedford MR, Penn CW et al., 2014. Extensive microbial and functional diversity within the chicken caecal microbiome. PLoS One, 9: e91941, doi: 10.1371/ journal.pone.0091941

Suzuki K and Nakajima A, 2014. New aspects of IgA synthesis in the gut. Int Immunol, 26(9): 489-494, doi: 10.1093/intimm/dxu059

Tanca A, Fraumene C, Manghina V, Palomba A, Abbondio M et al., 2017. Diversity and functions of the sheep faecal microbiota: A multi- omic characterization. Microb Biotechnol, 10: 541554, doi: 10.1111/1751- 7915.12462

Tizard IR, 2017. Veterinary Immunology-E-Book. Elsevier Health Sciences

Turner PV, 2018. The role of the gut microbiota on animal model reproducibility. Anim Models Exp
Med, 1(2): 109-115, doi: 10.1002/ame2.12022

Uyeno Y, Sekiguchi Y and Kamagata Y, 2010a. rRNAbased analysis to monitor succession of faecal bacterial communities in Holstein calves. Lett Appl Microbiol, 51: 570-7, doi: 10.1111/j.1472765X.2010.02937.X

Uyeno Y, Sekiguchi Y, Tajima K, Takenaka A, Kurihara $\mathrm{M}$ et al., 2010b. An rRNA-based analysis for evaluating the effect of heat stress on the rumen microbial composition of Holstein heifers. Anaerobe, 16(1): 27-33, doi: 10.1016/ j.anaerobe.2009.04.006

Wang J, Chen L, Zhao N, Xu X, Xu Y et al., 2018. Of genes and microbes: solving the intricacies in host genomes. Protein Cell, 9(5): 446-461, doi: 10.1007/s13238-018-0532-9

Wei S, Morrison M and Yu Z, 2013. Bacterial census of poultry intestinal microbiome. Poult Sci, 92 (3): 671-683, doi: 10.3382/ps.2012-02822

Xu X, Xu P, Ma C, Tang J and Zhang X, 2013. Gut microbiota, host health, and polysaccharides. Biotechnol Adv, 3: 318-337, doi: 10.1016/ j.biotechadv. 2012.12.009

Yegani M and Korver DR, 2008. Factors affecting intestinal health in poultry. Poult Sci, 87: 20522063, doi: 10.3382/ps.2008-00091

Yildirim S, Yeoman CJ, Sipos M, Torralba M, Wilson BA et al., 2010. Characterization of the fecal microbiome from non-human wild primates reveals species specific microbial communities. PLoS One, 5: e13963, doi: 10.1371/journal. pone.0013963

Zhang A and Steen TY, 2017. Gut microbiomics - A solution to unloose the gordian knot of biological effects of ionizing radiation. J Hered, 109(2): 212221, doi: 10.1093/jhered/esx059

Zhong Z, Zhou G, Yang L, Liu H and Song W, 2014. The biomass allocation patterns of desert shrub vegetation in the Qiadam Basin, Qinghai. China J Desert Res, 34: 1042-1048

Zhou W, Wang Y and Lin J, 2012. Functional cloning and characterization of antibiotic resistance genes from the chicken gut microbiome. Appl Environ Microbiol,78:3028-3032, doi:10.1128/AEM. 06920-11 